\title{
Ophthalmological and obstetric management in pregnant women with retinal disorders
}

\author{
Joanna Moneta-Wielgos ${ }^{1}$, Michal Lipa ${ }^{2}$, Joanna Brydak-Godowska ${ }^{3}$, \\ Marek Rekas ${ }^{1}$, Miroslaw Wielgos ${ }^{2}$ \\ ${ }^{1}$ Department of Ophthalmology, Military Institute of Medicine, Warsaw, Poland \\ ${ }^{2} 1$ st Department of Obstetrics and Gynecology, Medical University of Warsaw, Poland \\ ${ }^{3}$ Department of Ophthalmology, Medical University of Warsaw, Warsaw, Poland
}

\begin{abstract}
Objectives: To analyze the clinical significance of ophthalmological assessment in pregnant women affected with degenerative retinal lesions, and the lesions' clinical relevance in determining the obstetric management and delivery method.

Material and methods: 69 pregnant women affected with retinal degenerative lesions were included in our study. In each patient, the risk of ophthalmological complications during vaginal delivery was evaluated. After the woman's delivery, alignment between the ophthalmological recommendations and the obstetric management were analyzed. Each case where the management plan differed from the clinical proceedings was thoroughly investigated to determine the cause.

Results: In 69 pregnant women the risk of ophthalmological complications was evaluated, and in 24 cases (35\%) assessed as low, as medium in 37 cases (54\%) and as high in 8 cases (11\%). Among the 69 patients, 42 of women delivered vaginally and the remaining 27 underwent caesarean section. In the high-risk group, the rate of caesarean section was $87 \%$, while in both the low- and medium-risk groups the rate of vaginal births was $75 \%$. Two years of postnatal ophthalmological follow-up did not reveal any complications that could have been associated with the delivery.

Conclusions: Every pregnant woman should undergo ophtalmological examination to assess peripartum risk of complications and determine the method of delivery.
\end{abstract}

Key words: myopia; ophtalmological examination; caesarean section; laser photocoagulation

\section{INTRODUCTION}

The presence of peripheral, degenerative lesions in the retina remains one of the most important risk factors of ophthalmological complications, most notably, retinal detachment. Pathological retinal lesions may be congenital or acquired, and either located in the central or peripheral parts. In some cases, they may involve retinal vessels as well [1]. The most frequent ophthalmological issue during pregnancy remains myopia associated with peripheral degenerative lesions and the risk of retinal detachment [2]. However, in myopic patients with degenerative lesions, retinal detachment is caused by pathology in the vitreous rather than in the retina itself [3]. It is noteworthy that most cases of peripheral lesions in pregnant patients are detected coincidentally. Unfortunately, patients tend to avoid laser photocoagulation during gestation due to fears for the wellbeing of the fetus, about the potential side effects of the intervention, or its impact on the mode of the delivery. Nonetheless, pregnancy is not a contraindication for ophthalmological treatment and every patient diagnosed with peripheral retinal lesions should undergo laser photocoagulation to separate degenerative tissues from the normal retina [4]. According to a consensus among obstetric and ophthalmological recommendations in 2017 , laser photocoagulation should be performed at least 4 weeks before the estimated date of delivery to significantly reduce the risk of intrapartum ophthalmological complications [5]. Prior to delivery, the level of risk of ophthalmological complications for vaginal delivery cases may be assessed according to a three-degree severity scale:

- low

- medium

- high 
The low-risk group is defined as having no contraindications for vaginal delivery. On the other hand, in the high-risk category of ophthalmological complications, elective caesarean section should be recommended. In the medium-risk group, the possibility of eyesight deterioration still exists, however it seems that vaginal delivery remains safe. In this group, shortening the second phase of the delivery with vacuum or forceps may be considered; however, these procedures are relatively rare in contemporary obstetrics.

\section{Aim of the study}

To analyze the clinical significance of ophthalmological assessment in pregnant women affected with degenerative retinal lesions, and the lesions' clinical relevance in determining the obstetric management and delivery method.

\section{MATERIAL AND METHODS}

69 pregnant women diagnosed with degenerative retinal lesions during ophthalmological examinations were included in our study. Lesions were revealed in 121 eyes. Each patient underwent an ophthalmological assessment of the risk of peripartum complications arising during vaginal delivery and each woman was assigned to one of the low-, medium- or high-risk groups. In addition, we analyzed laser photocoagulation throughout the pregnancy or refractive eye surgery in the past. In each case where the method of delivery was different from that of the ophthalmological recommendations we carefully investigated what happened to identify the specific indications.

\section{RESULTS}

Of the 69 pregnant women in our study, 24 patients were assigned to the low-risk group (35\%), 37 to the medium-risk group (54\%) and only 8 to the high-risk group (11\%). Overall, the rate of vaginal delivery was $61 \%$ compared with $39 \%$ for caesarean section.

In high-risk group, 7 patients (87\%) underwent caesarean section and only 1 (13\%) delivered naturally due to lack of consent for a caesarean section. Out of 37 patients in the medium-risk group, 14 underwent caesarean section (38\%) while 23 delivered naturally (62\%). Among the medium-risk patients who underwent caesarean section the indications for an operative delivery were: patients' concerns about peripartum ophthalmological complications (50\%), obstetricians' concerns about the increased risk of ophthalmological complications (29\%), and in 3 cases (21\%) caesarean section was performed because of strictly obstetric indications. On the other hand, among 24 patients with a low-risk of ophthalmological complications, 18 delivered naturally (75\%) and 6 patients underwent caesarean section due to their concerns about peripartum ophthalmological complications.
From among the whole study group, 43 patients qualified for laser photocoagulation during pregnancy. After the laser procedure their risk was reassessed, with the results that despite the treatment, from 4 patients in the high-risk group (9\%), 3 women underwent caesarean section and 1 delivered naturally due to there being no consent for operative delivery. In 6 patients, the risk of ophthalmological complication was assessed as low (14\%), and 5 of them delivered naturally while 1 delivered by caesarean section because of obstetric indications. Most of the patients who underwent laser photocoagulation were assessed as a medium risk for ophthalmological complications (77\%). Among this group there were 20 natural deliveries (70\%) and 13 operative deliveries. In the subgroup of intermediate-risk, 4 patients qualified for caesarean section based on obstetricians' concerns, 6 cases because of patients' concerns, and 3 cases because additional obstetric factors occurred.

In addition, in our study group there was a group of 7 patients who had undergone refractive surgery in the past. In 2 of the pregnant women who delivered naturally the risk was evaluated as low (29\%), in 4 as intermediate (49\%) and in 1 as high (14\%). Of the intermediate- and high-risk groups only 1 patient delivered naturally, and in the remaining 4 cases caesarean section was performed because of obstetricians' concerns.

During two years of postpartum follow-up there were no complications that could have been associated with delivery.

\section{DISCUSSION}

The rate of caesarean sections worldwide is growing and has been referred to a"plague" in contemporary obstetrics [6]. Unfortunately, the trend is evident in Poland, as well. In 2016, $43 \%$ of all deliveries were concluded by caesarean section [7]. Despite epidural anesthesia and the improving quality of perinatal care, caesarean section continues to be associated with increased risks of both maternal and neonatal complications during delivery [8-10]. On the other hand, when the incidence of caesarean section rises above $20 \%$ in the population it is not matched by a corresponding decrease in maternal or neonatal morbidity. The most frequent indications for operative delivery are obstetric ones, however so called non-obstetric indications are also frequent [11, 12]. For many years, the ophthalmological indications for caesarean section were not summarized in any scientific associations' published recommendations. However, in the current situation, there has been a significant improvement thanks to an obstetric and ophthalmological consensus regarding the mode of the delivery in patients with eye disorders that was published in 2017 [5]. Thanks to this publication, those ophthalmological disorders that may be regarded as an indication for caesarean section were precisely identi- 
fied. Nowadays, cooperation between ophthalmologists and obstetricians allows practitioners to assess the risk of peripartum complications and to define the appropriate mode of the delivery. It is worth noting that suggestions that eyesight may worsen permanently after delivery, has not been borne out in the studies.

As refractive surgery in patients who are affected with myopia became a frequent procedure in the general population and as many women no longer use lenses or glasses, every pregnant patient should be interviewed by their obstetrician about their past medical history to evaluate the risk of intrapartum ophthalmological complications. Most patients are not aware that though refractive surgical procedures increase the acuteness of vision, they do not however, prevent ophthalmological complications occurring during delivery. Moreover, refractive surgery does not affect the state of retina and other potential pathologies associated with myopia such as glaucoma or dregs in the corpus vitreum. Due to refractive surgery, the thickness of the cornea is reduced, and every patient should undergo pachymetry to assess their corneal thickness after the procedure. The normal central corneal thickness (CCT) across the population is 510-570um. According to the previously mentioned consensus, in cases where the corneal thickness is less than 350 um elective caesarean section should be considered because of the high risk of corneal complications. Separation of the layers of the Descemet membrane or corneal ectasia may cause permanent deterioration in the eyesight and irregular astigmatism. In such cases surgical treatment may be needed [13]. Every patient with a history of ophthalmological complications, e.g. high myopia, diabetic retinopathy, after eye surgery, keratoconus and visual acuity disorders should be examined by an experienced ophthalmologist during the first trimester of the pregnancy. The physician may assess the risk of peripartum complications and the potential need for treatment during the course of the pregnancy [14].

Our analysis shows that most patients with a high risk of ophthalmological complications underwent caesarean section (almost in 90\%). On the other hand, over $75 \%$ of the patients in the low-risk group delivered naturally when no additional obstetric indications for caesarean section occurred. Nonetheless, the most challenging group, from a clinical perspective were those women with a medium risk of ophthalmological complications. Among those patients, the rate of vaginal compared with operative deliveries were similar ( $40 \%$ vs $60 \%$, respectively). The most frequent indications for caesarean section were obstetricians' or patients' concerns about natural delivery. However, during two years of postnatal follow up with the whole study group, no eye complications occurred that could have been associated with delivery. This fact suggests that in the group of subjects with a medium risk of ophthalmological complications, there may be an unnecessary rise in the number of elective caesarean sections due to either obstetricians' or patients' concerns. Our data suggest that three-degree scale of risk should be revaluated and most likely modified. Recalibrating the risk scale into two risk groups, namely into low-risk and high-risk, may prevent clinical predicaments and facilitate better obstetric management. This variant seems to be clearer and more applicable in clinical practice, wherein low-risk patients may be qualified for vaginal delivery while high-risk patients should be scheduled for elective caesarean section.

\section{CONCLUSIONS}

1. Most patients with a high risk of peripartum complications, caesarean section was the main mode of delivery, while in the low-risk group most patients delivered naturally.

2. In medium-risk group rates of natural compared with operative deliveries were similar ( 40 vs $60 \%$, respectively).

3. Prenatal laser photocoagulation increases the chances for a vaginal delivery.

4. The main cause of obstetric management that differs from the ophthalmological recommendations were the result of the cautious attitudes of obstetrician attitude and patients' concerns about peripartum complications.

5. Two-degree risk scale seems to be more useful in clinical practice than three-degree scale which is currently in use by ophtalmologists.

\section{REFERENCES}

1. Bruce A, O'Day J, McKay D, et al. Myopic degeneration: Pathological myopia. The Optician. 2007; 234(43).

2. Landau $D$, Seelenfreund $M$, Tadmor $O$, et al. The effect of normal childbirth on eyes with abnormalities predisposing to rhegmatogenous retinal detachment. Graefe's Archive for Clinical and Experimental Ophthalmology. 1995; 233(9): 598-600, doi: 10.1007/bf00404712.

3. Dragoumis I, Richards A, Alexander $P$, et al. Retinal detachment in severe myopia. Lancet. 2017; 390(10090): 124, doi: 10.1016/S01406736(17)31614-8, indexed in Pubmed: 28699591.

4. Dragoumis I, Richards A, Alexander $\mathrm{P}$, et al. Retinal detachment in severe myopia. Lancet. 2017; 390(10090): 124, doi: 10.1016/S01406736(17)31614-8, indexed in Pubmed: 28699591.

5. Konsensus okulistyczno-położniczy w sprawie wskazań do rozwiązania porodu drogą cięcia cesarskiego z powodu zmian w narządzie wzroku. Polskie Towarzystwo Okulistyczne Warszawa, 09. ; 02: 2017.

6. World Health Organization Human Reproduction Programme, 10 April 2015. WHO Statement on caesarean section rates. Reprod Health Matters. 2015; 23(45): 149-150, doi: 10.1016/j.rhm.2015.07.007, indexed in Pubmed: 26278843.

7. https://prog.nfz.gov.pl/app-jgp/Start.aspx

8. Cantwell R, Clutton-Brock T, Cooper G, et al. Saving Mothers' Lives: Reviewing maternal deaths to make motherhood safer: 2006-2008. The Eighth Report of the Confidential Enquiries into Maternal Deaths in the United Kingdom. BJOG. 2011; 118 Suppl 1: 1-203, doi: 10.1111/j.1471-0528.2010.02847.x, indexed in Pubmed: 21356004.

9. Creanga AA, Bateman BT, Butwick AJ, et al. Morbidity associated with cesarean delivery in the United States: is placenta accreta an increasingly important contributor? Am J Obstet Gynecol. 2015; 213(3): 384.e1384.11, doi: 10.1016/j.ajog.2015.05.002, indexed in Pubmed: 25957019.

10. Berthelot-Ricou A, Lacroze V, Courbiere B, et al. Respiratory distress syndrome after elective caesarean section in near term infants: a 5-year cohort study. J Matern Fetal Neonatal Med. 2013; 26(2): 176-182, doi: 10.3109/14767058.2012.733743, indexed in Pubmed: 23013109. 
11. Spong $C Y$, Berghella $V$, Wenstrom $K D$, et al. Preventing the first cesarean delivery: summary of a joint Eunice Kennedy Shriver National Institute of Child Health and Human Development, Society for Maternal-Fetal Medicine, and American College of Obstetricians and Gynecologists Workshop. Obstet Gynecol. 2012; 120(5): 1181-1193, doi: http://10.1097/AOG.0b013e3182704880, indexed in Pubmed: 23090537.

12. Groen RS, Trelles M, Caluwaerts S, et al. A cross-sectional study of indications for cesarean deliveries in Médecins Sans Frontières facilities across 17 countries. Int J Gynaecol Obstet. 2015; 129(3): 231-235, doi: 10.1016/j.ijgo.2014.12.008, indexed in Pubmed: 25770352.

13. Keep an eye on standardized management of laser refractive surgery under its rapid development. 2018; 54(10): 721-725.

14. Pallasmaa N, Ekblad U, Aitokallio-Tallberg A, et al. Cesarean delivery in Finland: maternal complications and obstetric risk factors. Acta Obstet Gy necol Scand. 2010; 89(7): 896-902, doi: 10.3109/00016349.2010.487893, indexed in Pubmed: 20583935. 